\title{
Arthroscopy of the Pediatric Elbow: Review of the Current Concepts
}

\section{Artroscopia del codo pediátrico: revisión de los conceptos actuales}

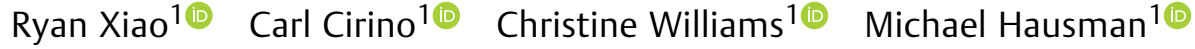 \\ ${ }^{1}$ Leni and Peter W. May Department of Orthopedic Surgery, Icahn \\ School of Medicine at Mount Sinai, New York, New York, United Sates \\ Address for correspondence Ryan Xiao MD, 5 E 98th St, 9th FI, New \\ York, NY, 10029, United Sates (e-mail: ryan.xiao@mountsinai.org).
}

Rev Iberam Cir Mano 2021;49:56-65.

\begin{abstract}
As surgeons have become more familiar with elbow arthroscopy, the indications for arthroscopy of the pediatric elbow have expanded to include contracture releases, fracture fixation, treatment of osteochondritis dissecans (OCD) lesions, correction of elbow deformity, and debridement of soft tissue and bony pathologies. The treatment of various pathologies via an arthroscopic approach demonstrates equal, if not better, efficacy and

\section{Keywords}

- pediatric

- elbow

- arthroscopy

- osteochondritis dissecans

- lateral condyle

\section{Resumen}

$$
\begin{aligned}
& \text { Palabras clave } \\
& \text { - pediátrico } \\
& \text { - codo } \\
& \text { - artroscopia } \\
& \text { - osteocondritis } \\
& \text { disecante } \\
& \text { - cóndilo lateral }
\end{aligned}
$$
safety as open surgery for the pediatric elbow. Arthroscopy provides the unique advantage of enabling the performance of extensive surgeries through a minimally-invasive approach, and it facilitates staged interventions in cases of increased complexity. For fracture work, arthroscopy enables direct visualization to assess reduction for percutaneous fixations. While future research is warranted to better evaluate the indications and outcomes of pediatric elbow arthroscopy, this update article presents a review of the current literature, as well as several innovative cases highlighting the potential of arthroscopy.

A medida que los cirujanos se han familiarizado con la artroscopia del codo, las indicaciones para la artroscopia del codo pediátrico se han ampliado para incluir la liberación de contracturas, la fijación de fracturas, el tratamiento de lesiones de osteocondritis disecante (OCD), la corrección de la deformidad del codo, y el desbridamiento de patologías óseas y de tejidos blandos. El tratamiento de diversas patologías mediante un abordaje artroscópico demuestra la misma eficacia y seguridad, si no mejor, que la cirugía abierta del codo pediátrico. La artroscopia proporciona la ventaja única de permitir la realización cirugías extensas a través de un abordaje mínimamente invasivo, y facilita las intervenciones por etapas en casos de mayor complejidad. Para las fracturas, la artroscopia permite la visualización directa para evaluar la reducción de las fijaciones percutáneas. Si bien se justifica la investigación futura para evaluar mejor las indicaciones y los resultados de la artroscopia del codo pediátrico, este artículo de actualización presenta una revisión de la literatura actual y varios casos innovadores que destacan el potencial de la artroscopia.
\end{abstract}

received

March 3, 2021

accepted

March 8, 2021
DOI https://doi.org/ $10.1055 / \mathrm{s}-0041-1730394$ ISSN 1698-8396.

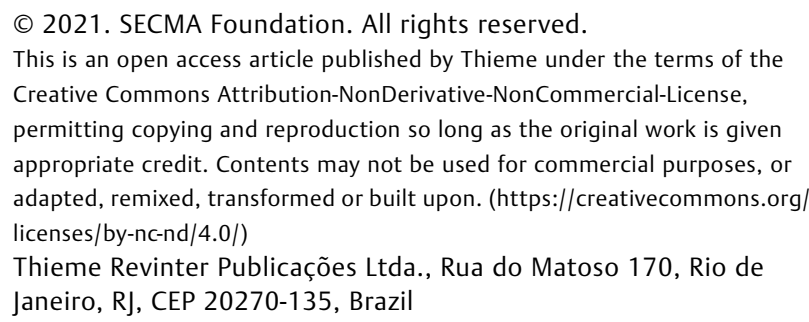




\section{Introduction}

Arthroscopy provides a minimally-invasive method to treat both acute and chronic conditions, but has historically been a challenge in the elbow due to the complex articular anatomy, confined joint space, and proximity of neurovascular structures. Historically, fear of complications with arthroscopy has limited its appeal and application to pediatric elbows. ${ }^{1}$ However, recent studies ${ }^{2-5}$ have demonstrated low complication rates in pediatric elbow arthroscopy, ranging from $0 \%$ to $8 \%$ and comparable to the $5 \%$ to $11 \%$ complication rate reported in adult elbow arthroscopic procedures. Examining complication rates after elbow arthroscopy in a large cohort of adult and pediatric patients, Intravia et al. ${ }^{6}$ reported no significant difference in the incidence of nerve palsy, heterotopic ossification, or infection between adult and pediatric arthroscopic procedures.

Advancements in instrumentation and technique have significantly expanded the pathologies treatable via arthroscopy, with pediatric elbows as one of the greatest beneficiaries. As with any procedure, surgical success depends on achieving good exposure and minimizing collateral damage. Arthroscopy is our preferred approach where possible, as it can increase surgical precision due to magnification; decrease the risk of peripheral damage, pain, and scar-tissue formation; and is amenable to staged procedures. One issue, however, is that, while pediatric orthopedists are experienced in the treatment of children and adolescents, they may lack proficiency in arthroscopy. This provides an opportunity for collaboration among orthopedists.

Though initial interest in pediatric elbow arthroscopy focused largely on the treatment of osteochondritis dissecans (OCD) lesions, the role of arthroscopy has expanded to include contracture release, arthroscopic-assisted fracture fixation, debridement of bony and soft tissue pathologies, correction of bony deformity, and release of synostosis. $2,7-10$ While its complication profile appears to be improved from that of open elbow surgery, elbow arthroscopy is a challenging procedure with a steep learning curve., ${ }^{5,11}$ The minimally-invasive nature of arthroscopy is of particular utility in the pediatric population, given the ability to avoid the morbidity of large open incisions. The present update article aims to address the current state of pediatric elbow arthroscopy and demonstrate several innovative cases highlighting the versatility of arthroscopy in treating pathologies of the pediatric elbow.

\section{Patient Set-up and Arthroscopy Portals}

In general, our preference is for regional anesthesia, with a preoperative motor and sensory block via an indwelling catheter, which allows for immediate control of the postoperative pain and rehabilitation as needed. If there is concern for compartment syndrome, a regional block should be avoided. While elbow arthroscopy can be performed in the prone, lateral decubitus, or supine positions, our preference is supine, with the operative arm draped freely and a tourniquet placed high on the upper brachium. The utiliza- tion of an Articulated Sterile Intraoperative Positioning System (ASIP, McConnell Orthopedic Manufacturing Co., Greenville, TX, US) enables ample shoulder and elbow positioning without obstructing operative maneuvers. In cases in which the patient's arm is too small for a secure fit within the ASIP, a surgical assistant is required to hold the limb.

In adolescent and larger children, a standard $30^{\circ}, 4.5-\mathrm{mm}$ shoulder arthroscope and associated electrocautery wand, shaver, and burr can be utilized; however, younger children ( $\sim 5$ years of age or younger) may require a $2.5-\mathrm{mm}$ wrist arthroscopy set-up. Distension of the joint with saline will displace the capsule and neurovascular structures anteriorly to aid in safe portal creation. In the setting of trauma, an effusion may already be present and sufficient. Pump pressure should be set to a maximum of $25 \mathrm{mmHg}$, and inflow should run solely through the tip of the trocar sheath, as opposed to more standard side-fenestrated trocars. Outflow is managed through the use of an arthroscopic shaver. Fluid management is of utmost importance to avoid extravasation and subsequent swelling, which can alter landmarks for portal placement.

Portal placement is similar to that used in adults, and knowledge of the surgical anatomy is necessary to avoid the multiple nearby neurovascular structures. Bony landmarks, including the medial and lateral epicondyle, the tip of the olecranon, the radial head, and the posterior radiocapitellar joint are reliable and reproducible for portal localization, although some may be cartilaginous depending on the age of the child. Yoo et al. ${ }^{12}$ demonstrated that condylar width and body mass index were correlated with proximal anterior capsule location. Prior trauma, heterotopic ossification, or other distortions of normal anatomy are relative contraindications to elbow arthroscopy. The ulnar nerve should be palpated through flexion and extension to ensure there is no subluxation that would place it at risk during the creation of the anteromedial portal. Although uncommon in children, a prior ulnar nerve transposition is a contraindication to elbow arthroscopy if it cannot be clearly palpated. In the case of long-standing contracture, an in-situ ulnar nerve release is warranted before starting the procedure.

Commonly used portals include the transtricipital, posterolateral, midlateral (soft-spot), anteromedial, and anterolateral portals. Accessory portals are utilized as required by the pathology or surgical procedure. In our experience, placement of the posterior portal should begin with the transtricipital portal and anteriorly with the anteromedial portal to enable direct visualization of the anterolateral portal. $^{7,13}$ - Fig. 1 shows the common elbow arthroscopy portals.

\section{Release of Elbow Contracture}

Historically, the results of open elbow contracture release in pediatric patients are not as favorable as those achieved in adults. ${ }^{14,15}$ While the reasons are not entirely clear, one frequent observation is that the child's nerves never reached adult length due to the contracture, and injury during periods of rapid growth will quickly result in the nerve being 
A

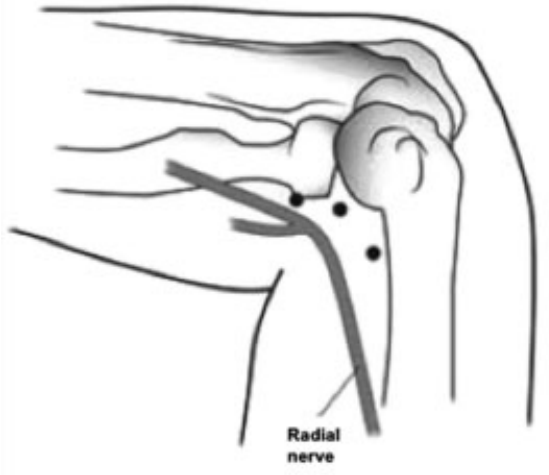

B

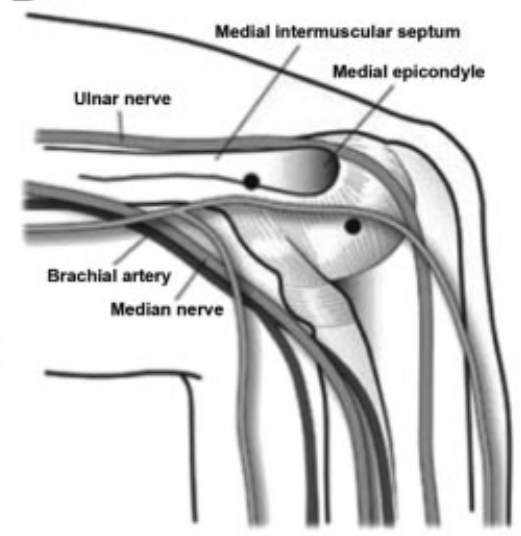

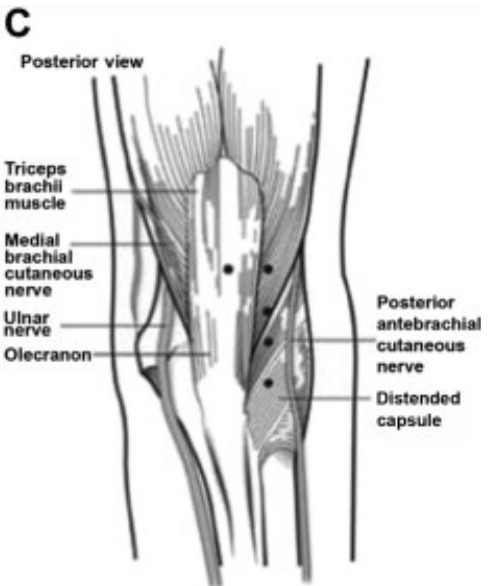

Fig. 1 Common Portals for Elbow Arthroscopy. (A) Lateral portals: proximal anterolateral, anterior radiocapitellar, posterior radiocapitellar. (B) Medial portals: proximal anteromedial and accessory anteromedial. (C) Posterior portals: transtricipital, proximal posterolateral, distal posterolateral, and accessory posterolateral. (Reprinted with permission from Koehler et al. ${ }^{21}$ )

excessively tensioned with extension. Thus, earlier intervention may minimize this problem by preventing nerve contracture. This is rarely, if ever, encountered in adults whose contracture-causing injury occurred after skeletal maturity. While we lack sufficient evidence to fully support this hypothesis, our early experience is suggestive. Arthroscopic release or even staged arthroscopic release are more acceptable and tolerated by parents and children, thus allowing earlier and, if necessary, repeated intervention to prevent traction on the nerves and thus improve the outcomes for contracture release.

As with adult arthroscopic contracture releases, special care must be taken to avoid the neurovascular structures, which can easily be accidentally injured if the arthroscopic shaver is directed toward the elbow capsule during debridement. Our preferred technique can be found in the October 2018 issue of the Journal of Pediatric Orthopedics. ${ }^{19}$

The common causes of pediatric elbow contractures are similar to those of the adult population, and include posttraumatic sequelae, muscular imbalance, congenital dislocations, burns, inflammatory arthropathies, hemophilia, and sepsis. The variety of etiologies and small patient cohorts make direct comparisons difficult, and few studies exist in the pediatric literature regarding arthroscopic release of elbow contracture. A 2013 systematic review of 798 predominantly adult subjects by Ködde et al. ${ }^{16}$ found that arthroscopic contracture releases demonstrated results similar to those of open procedures with less morbidity and lower complication rates. The published literature on the release of pediatric arthroscopic elbow contracture, by comparison, comprises less than 50 patients in total, but does report similar findings. ${ }^{2,3,17-19}$

The first article to describe the use of elbow arthroscopy for contracture in a pediatric population was the one by Micheli et al. $^{2}$ (2001). In their series of 49 pediatric elbow arthroscopies, the authors performed 9 arthroscopic releases for arthrofibrosis and decreased range of motion. The majority of patients achieved good to excellent results based on the modified Andrews elbow score, with an average increase of $32^{\circ}$ of extension and $21^{\circ}$ of flexion at a minimum follow-up of 2 years. No statistical analysis was performed to accompany their results. They reported no complications, but cautioned that elbow arthroscopy is an acquired skill, with significant risk even in the hands of experienced arthroscopists.

The largest series to date was published by our senior author in the Journal of Pediatric Orthopedics in 2018, which comprised 29 contracture releases in 25 patients with an average follow-up of 15 months. ${ }^{19}$ The most common etiology of contracture was following radial head fractures $(n=9)$, which has been described ${ }^{20}$ to occur more frequently in intra-articular fractures. The average preoperative flexion-extension arc measured $93^{\circ}$ and improved to $128^{\circ}$ $(p=0.00002)$ following release. The modest improvement in pronosupination of $12.2^{\circ} \pm 35.2^{\circ} \quad(p=0.097)$ can be explained by the majority of cases presenting primarily with pure flexion-extension contractures. In a subgroup analysis of 10 patients with significant limitations in pronosupination, a net improvement of $41^{\circ}$ was observed. Seven patients experienced a postoperative complication, including five minor complications (surgical site infection, two transient neuropraxias, persistent wound drainage, and recurrence of a prior physeal bar) and two major complications (supracondylar stress fracture through a distal humeral osteoplasty, and a recurrent contracture secondary to patient non-compliance). This study demonstrated an important equivalence to open pediatric elbow contracture releases as a less invasive alternative, which is susceptible to staging of complex pathologies.

\section{Fracture Fixation}

Though lateral condyle fractures (LCFs) are the most common fracture addressed arthroscopically in the pediatric population, the orthopedic literature also describes techniques for fixation of coronoid, supracondylar, medial epicondyle, radial head or neck fractures. $8,11,13,17,21-23$

Fractures of the lateral condyle are the second most common pediatric elbow fracture, and represent a diagnostic 


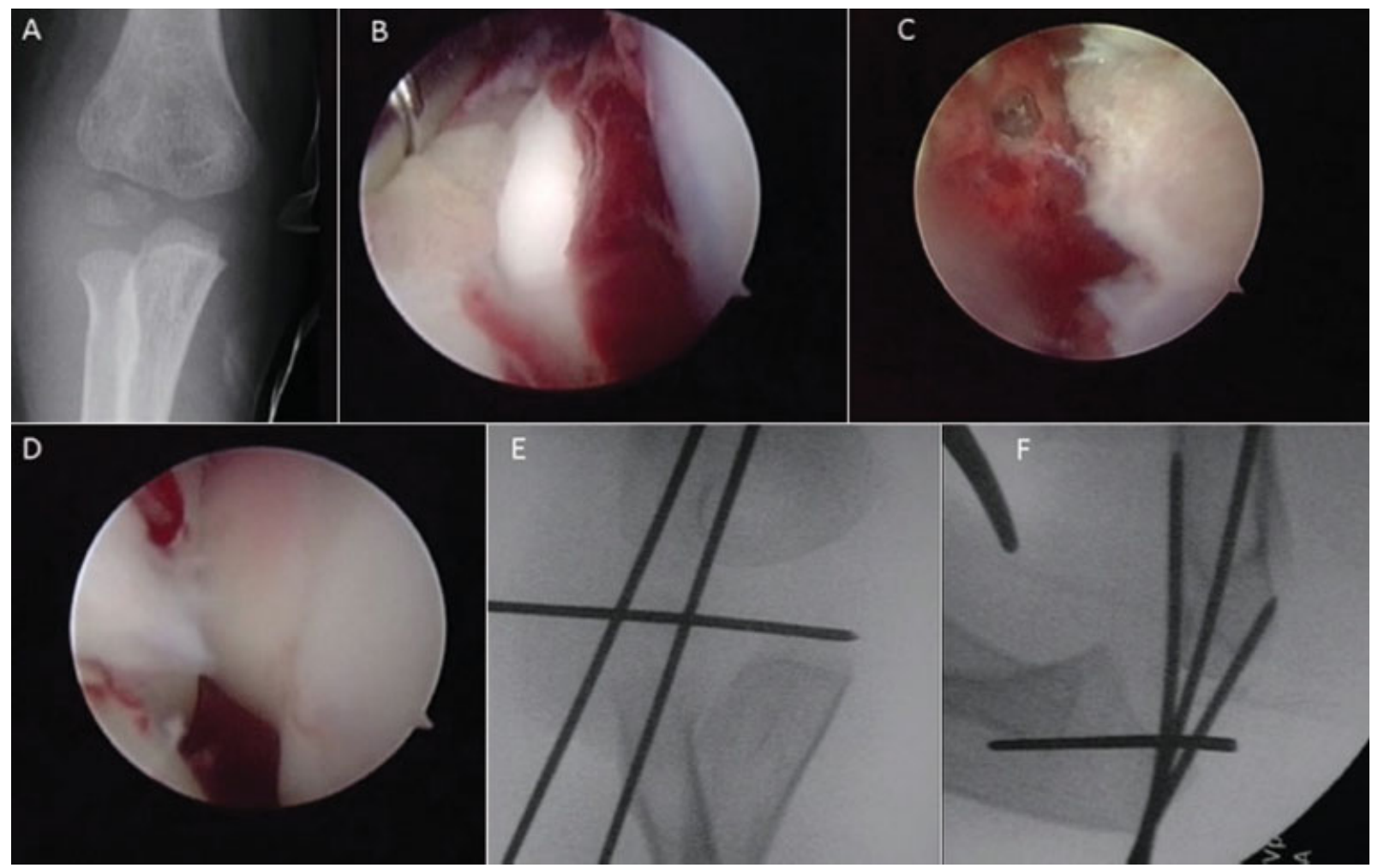

Fig. 2 5-year-old with a displaced lateral condyle fracture who underwent arthroscopic-assisted reduction and internal fixation. (A) Injury radiographs of the lateral condyle fracture. (B) Initial inspection from the proximal anteromedial portal demonstrates the fragment and fracture surface covered with hematoma. (C) The secondary ossification center is exposed, and a fixation pin is inserted under direct vision to ensure accurate placement. (D) The fracture is anatomically reduced, again under direct arthroscopic visualization. (EF) Kirschner wires are placed percutaneously to fix the fracture. The trajectory of the Kirschner wire is confirmed via fluoroscopy, and the maintenance of reduction is confirmed under arthroscopy.

and treatment challenge with a relatively high rate of severe complications, including avascular necrosis (AVN), non-union, growth arrest, subsequent deformity, and loss of motion. ${ }^{24,25}$ The limited ossification makes it difficult to correctly assess the type of fracture or level of displacement with radiographs alone. ${ }^{26}$ Even using intraoperative arthrography, one cannot be completely assured of either an anatomic reduction, as hematoma can prevent dye extravasation behind the displaced fragment, or fixation within the ossific nuclei. ${ }^{27}$

Arthroscopy is, essentially, a surgical approach (-Fig. 2). In the setting of LCFs, it affords a degree of exposure unachievable by open means without detaching critical ligaments and violating the precarious posterolateral blood supply that could cause AVN. It can be used diagnostically to assess the degree of fracture displacement as well as therapeutically to assist in debridement and achieving a stable, anatomic reduction.

Our preferred technique and results have been described in the October/November 2007 issue of the Journal of Pediatric Orthopedics, ${ }^{8}$ as have subsequent techniques by PerezCarro et al., ${ }^{22}$ Temporin et al., ${ }^{23}$ and Kang et al. ${ }^{11}$ In our series of 6 patients, with an average age of 4 years and treated within 24 hours of the injury, all went on to achieve full functional range of motion, with no statistical difference in range of motion or carrying angle compared with the uninjured contralateral elbow. One patient developed radiolucency of the capitellum without clinical significance. In the recent series by Kang et al., ${ }^{11}$ one-third of the patients undergoing closed reduction and percutaneous pinning (CRPP) required re-reduction after the arthroscopic assessment, despite an acceptable appearance on fluoroscopy. ${ }^{11}$ This may represent a patient at risk for complications if undergoing traditional CRPP alone. The authors noted full range of motion in 27/30 of patients, with the remaining 3 experiencing mild contractures $\left(10^{\circ}\right.$ to $20^{\circ}$ decrease regarding the opposite side). Complications included two transient radial nerve palsies and no instances of AVN or non-union.

Arthroscopic-assisted fracture management may be beneficial in other fractures about the elbow (coronoid, radial head/neck, capitellum, supracondylar), and recent small case series and reports have demonstrated good results with regard to fracture union, range of motion and complications. $^{7,21,28}$ There is also the potential for arthroscopic corrective osteotomies following supracondylar malunion, in which the current complication rates for open procedures is reported at upwards of $40 \%{ }^{29}$ Larger studies and trials are needed, however, to compare these minimally-invasive procedures to their open counterparts.

Clinical case 1: (- Fig. 2)

\section{Management of Osteochondritis Dissecans}

Another condition benefitting from the improved exposure that can be obtained with arthroscopy as well as the 


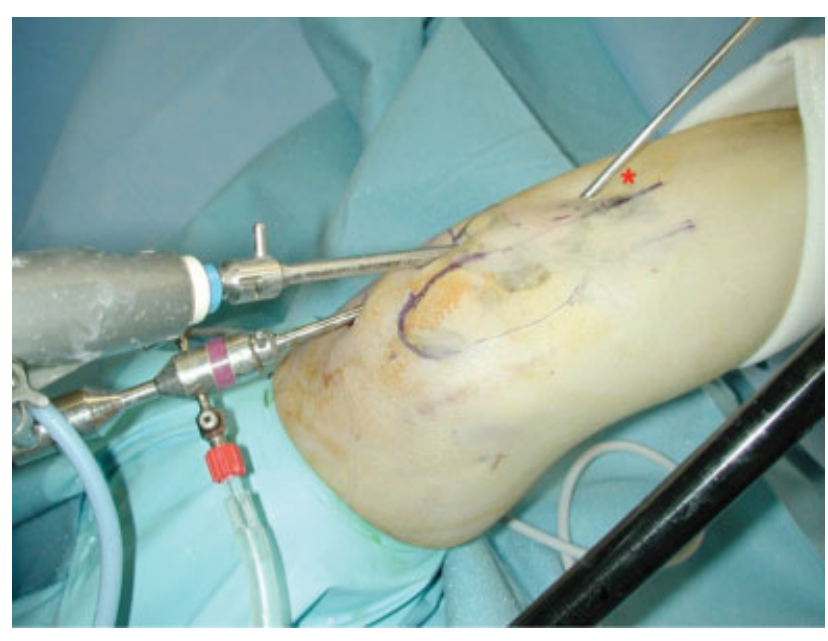

Fig. 3 The distal "para-ulnar" portal (marked with an asterisk) is placed well distal to the standard "soft-spot" portal.

increased precision of performing the procedure under magnification is OCD.

Osteochondritis dissecans is currently the most common indication for pediatric elbow arthroscopy. Multiple studies $^{2,30-33}$ in the orthopedic literature confirm its safety and efficacy. The majority of authors ${ }^{2,30,31}$ present chondroplasty, debridement of unstable lesions, and/or microfracture to address OCD lesions, though Tis et al. ${ }^{33}$ and Takeba et al. ${ }^{32}$ also report good short-term results after fixation of unstable OCD lesions with bioabsorbable pins. Miyake and Masotomi ${ }^{30}$ presented one of the largest series, including the surgical management of 106 OCD lesions in pediatric patients, and demonstrated overall good results after arthroscopic debridement and subchondral drilling; 99\% of patients returned to sport at an average of 2.4 months postprocedure, and $85 \%$ of patients returned to preinjury levels of sport. ${ }^{30}$ However, the authors caution that a reconstructive procedure such as an osteochondral autograft may better address large OCD lesions, given their poor outcomes in the subgroup of patients with large OCD lesions and an open radial head physis; they reported that all four such patients developed radial head enlargement, three went on to radiographic osteoarthritis of the radiohumeral joint, and two required radial head resection. ${ }^{30}$

When we address OCD lesions, standard portals are employed, with the exception of a distal "para-ulnar" portal (-Fig. 3) just radial to the lateral border of the ulna. The distal "para-ulnar" portal facilitates a trajectory that is perpendicular to the tangent to the capitellum at the site of the lesion, thereby enabling proper alignment in the setting of a reconstructive procedure like an osteochondral plug autograft or allograft.

The improved visualization facilitates more sophisticated repairs beyond simple drilling. These include stabilization of loose but undetached fragments, actual replacement and preservation of large fragments with subchondral drilling and bone grafting, cartilage repair in skeletally-immature patients, mosaicplasty, and treatment of trochlear lesions.
All of these procedures are greatly facilitated by arthroscopy, which, without releasing any ligaments, facilitates the exposure that is necessary not only to visualize the pathology, but also to pass the mattress-type sutures that are needed to adequately stabilize the fragments.

Our protocol also requires prolonged immobilization (usually 2 to 3 months) to allow for healing of the fragment or graft, which is confirmed by magnetic resonance imaging (MRI) or computed tomography (CT) scans. Initially, stiffness was a concern, but only one patient has required a subsequent arthroscopic release to regain full motion. Thus, performing these procedures with arthroscopic, rather than open, exposure may improve healing by allowing for longer periods of immobilization and protection without an increased risk of stiffness. Furthermore, if stiffness does occur, arthroscopic release can be performed.

Clinical case 2: (-Fig. 4)

Clinical case 3: (-Fig. 5)

\section{Deformity Correction}

Posttraumatic deformity correction may also be facilitated by arthroscopic techniques, which may be less painful and better accepted. The risk of physeal injury and malunion is a common complication following trauma to the pediatric elbow, including cubitus varus deformity following supracondylar fracture, and cubitus valgus deformity following lateral condyle non-union or malunion. ${ }^{34,35}$ While the sequelae are often largely issues of cosmesis (which by themselves are a common indication for a corrective procedure), we are becoming increasingly aware of the pain and functional limitation that can arise from resultant nerve palsies, $^{36}$ stiffness, and posterolateral rotatory instability. ${ }^{37}$ Additionally, malunion at the elbow may increase the risk of future fracture. ${ }^{38,39}$

Various osteotomies, including opening or closing wedge osteotomies, dome osteotomies, and distraction osteogenesis have been described to correct elbow deformities. ${ }^{40}$ However, open surgery for correction of pediatric elbow deformity is associated with a high complication rate, from $14 \%^{40}$ up to $40 \%^{34}$ in one series, including risks of nerve injury, insufficient correction due to loss of fixation, infection, growth arrest, stiffness, and unsightly scarring. ${ }^{29,34,40,41}$ Despite the prevalence of open procedures, the orthopedic literature remains scarce of arthroscopic techniques to address pediatric elbow deformity. Through an arthroscopic approach, we believe we can minimize the morbidity of deformity correction surgery while still achieving functional outcomes. Here, we present one case of correction of lateral condyle avascular necrosis and one case of correction of supracondylar malunion (-Figs. 6 and 7)

Clinical case 4: (-Fig. 6)

The patient's elbow motion is limited due to flattening of the fragmented humeral physis and the reciprocal changes in the radial head, which continues to grow and aggravate the deformity. Following failure of the non-operative 


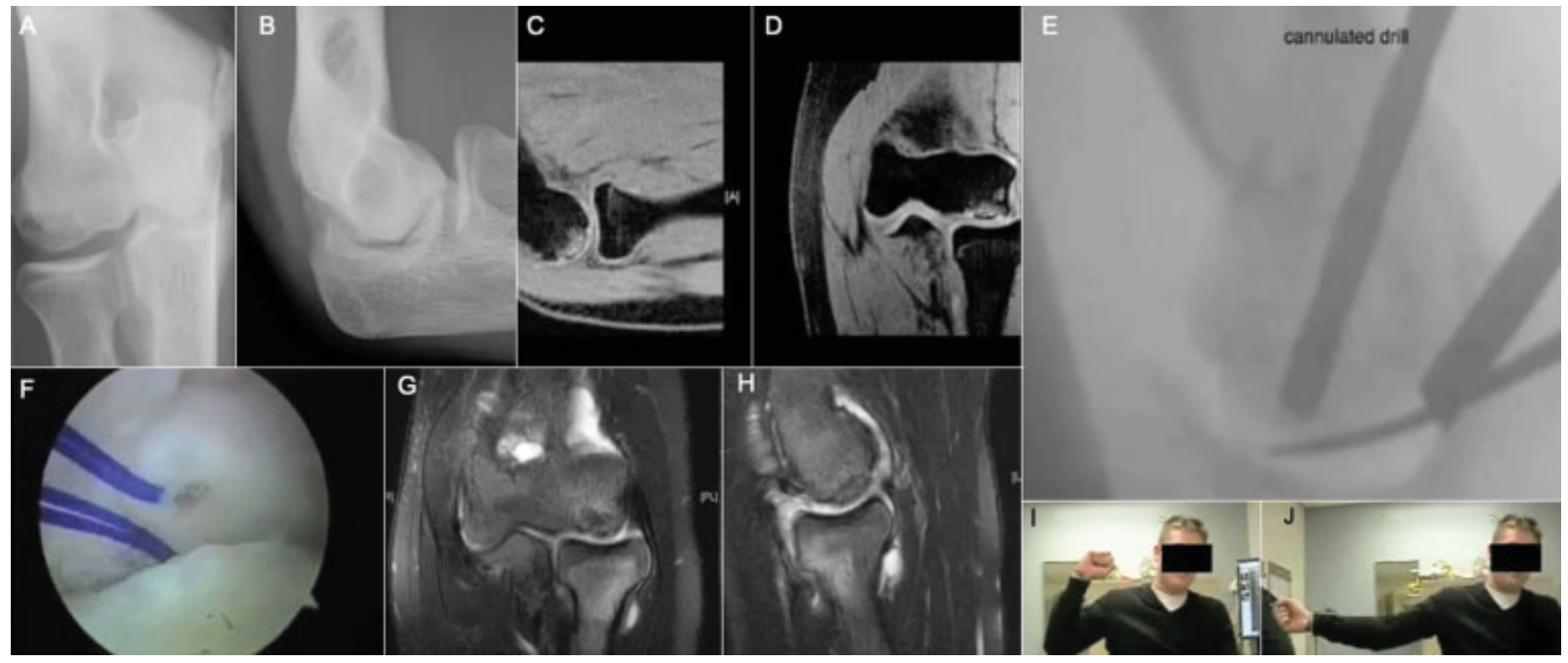

Fig. 4 13-year-old male patient with persistent elbow pain and failure of the conservative treatment. Via arthroscopy, his capitellum lesion was secured with suture and bone graft placed behind the OCD lesion. (A,B) Radiographs of an osteochondral lesion of the capitellum in a skeletallyimmature patient. (C,D) MRI correlation redemonstrating the capitellum lesion. The cartilaginous surface remains intact, and this was confirmed on arthroscopy. (E) A cannulated drill was placed into the center of the lesion up to but not through the subchondral bone, and placement was confirmed on fluoroscopy. A freer elevator was used to ensure that the lesion did not get displaced during drilling. (F) PDS (Ethicon, Somerville, $\mathrm{NJ}$, US) suture passed via a spinal needle through the center of the OCD lesion. Medial and lateral holes are created to fashion a mattress suture to secure the OCD lesion. Bone graft is used to backfill the cannulated drill path. $(\mathbf{G}, \mathbf{H})$ 3-month postoperative MRI demonstrating healing of the OCD lesion with bone bridging the formerly diseased capitellum. $(\mathrm{I}, \mathrm{J})$ Patient with almost complete elbow flexion and extension following the procedure.

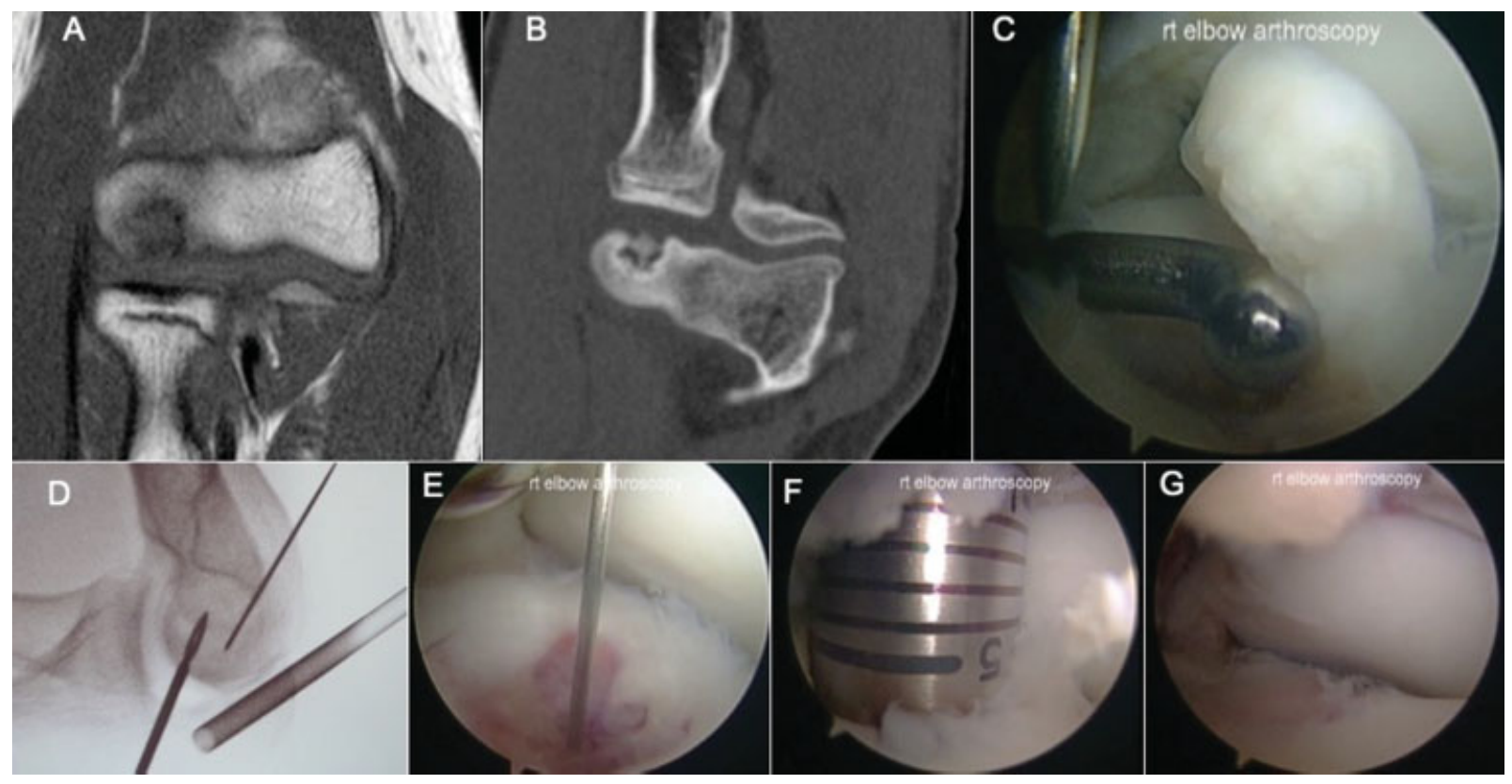

Fig. 5 Adolescent male with a large, unstable OCD lesion. As previously noted in the literature ${ }^{30}$, large and unstable OCD lesions often have poor results when debridement and chondroplasty alone are performed. This patient underwent an arthroscopic-assisted osteochondral autograft reconstruction, making use of the distal para-ulnar portal to obtain the necessary trajectory to secure the autograft into the osteochondral deficit. (A,B) MRI and CT scans demonstrating a large OCD lesion in the capitellum. (C) Arthroscopic probe demonstrating a large, grossly loose flap of the OCD lesion. (D,E) The center of the lesion was pinned, and a cannulated drill was passed over the Kirschner wire. Position was confirmed on fluoroscopy and arthroscopy. (F) Following subsequent reaming to prepare the recipient site, a sizer was used to measure and prepare the site for the autograft. $(G)$ The osteochondral autograft nicely fills the prior defect. No articular incongruency is noted. 


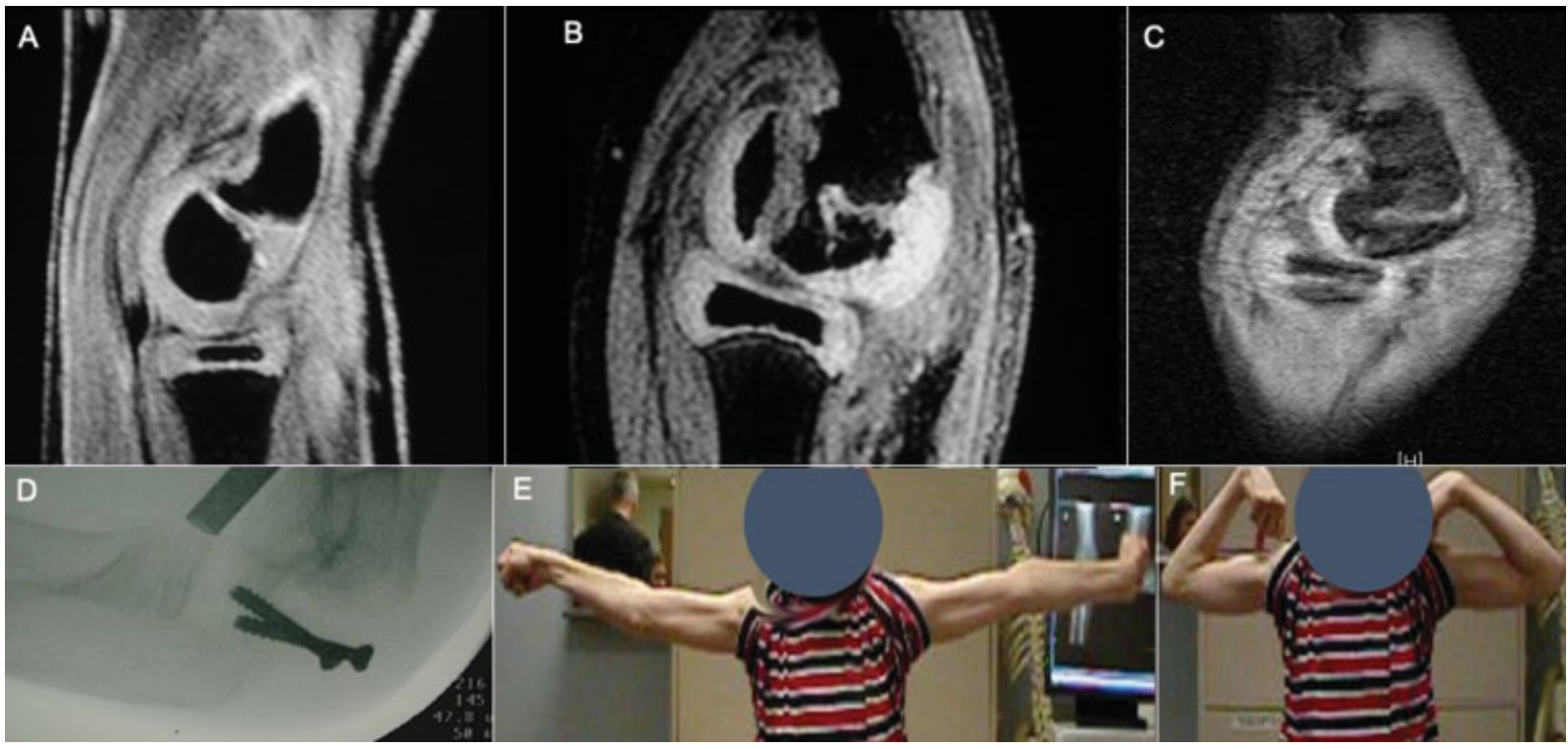

Fig. 6 8-year-old male with physeal fragmentation and deformity following a lateral condyle fracture of the elbow. (A) MRI of normal lateral condyle for comparison. (B) MRI of lateral condyle deformity and physeal bar with anterior humeral articular fragment. (C) MRI with improvement in the shape and contour of the lateral condyle following screw removal and healing. (D) Intraoperative fluoroscopy demonstrating restoration of a spherical capitellum through fixation of the anterior fragment with partially threaded posterior-to-anterior screws, physeal bar resection, and restoration of the normal anterior-to-posterior width of the lateral condyle. (E,F) Near-normal elbow flexion and extension following physeal correction. However, the physeal bar has reformed, so close, longitudinal observation is necessary.
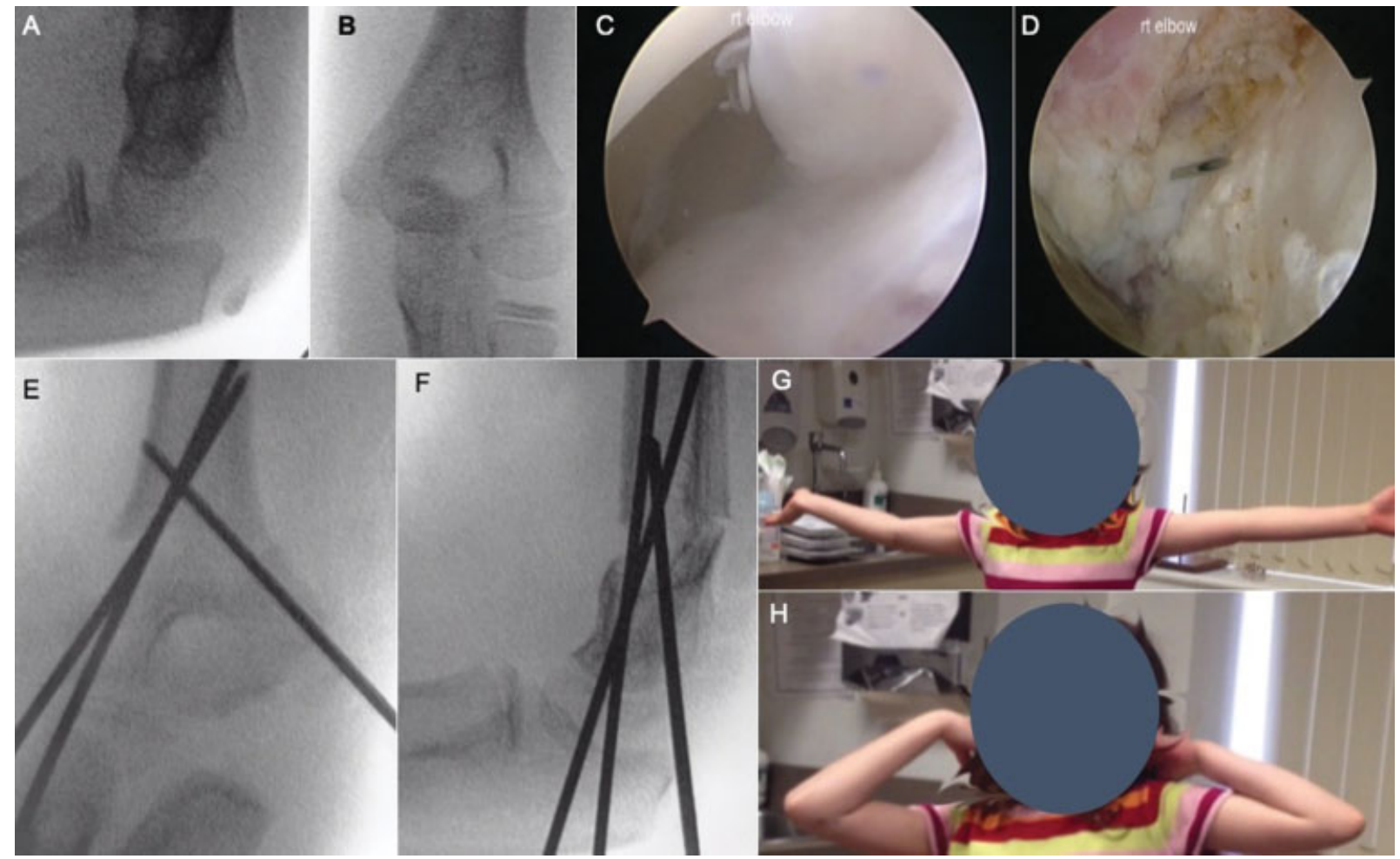

Fig. 7 7-year-old male with an extension malunion following a supracondylar elbow fracture. He had limited elbow flexion and was indicated for a supracondylar osteotomy for correction of his malunion. (A,B) Lateral and anteroposterior radiographs of this patient's extension malunion of prior supracondylar fracture. (C) Incongruent radiocapitellar motion with the radial head swiping across the capitellum during elbow flexion. (D) A needle is used to fluoroscopically confirm the location of the malunion so that an anatomic osteotomy can be performed. (E,F) Following manual osteoclasis of the posterior cortex, the distal humerus is flexed back into anatomic alignment and fixed with two lateral pins and one medial pin. The fracture is pinned and casted until healed. $(\mathrm{G}, \mathrm{H})$ Patient's range of motion 2 weeks after pin removal with near-normal elbow flexion and extension. 
management, he underwent resection of the bony physeal bar and arthroscopic-assisted internal fixation of the anterior articular fragment to the posterior humerus, similar to how we address adult capitellum fractures. Continued monitoring is needed to prevent radial overgrowth, but his condition and function were greatly improved following this procedure.

Clinical case 5: (-Fig. 7)

In this case, arthroscopic anteromedial and anterolateral portals were established. The apex of the deformity was visualized, and the location, confirmed on intraoperative fluoroscopy. The dimensions of the bony wedge resection were calculated preoperatively, and a burr was used to cut the anterior cortex. Holding the elbow in his/her hands, the surgeon used the thumbs to flex the distal fragment into the correct degree of flexion and the osteotomy was secured with Steinmann pins. These pins were subsequently removed following osteotomy healing.

In addition, the arthroscopic examination revealed an incongruent motion of the radial head across the capitellum. This was unexpected, and presented another compelling reason to correct the deformity and prevent future elbow pain and dysfunction. We believe that many elbow problems treated in adults, like premature arthrosis, may be the sequelae of childhood injuries that may be underrecognized on radiographic and clinical examination alone.

\section{Debridement of Soft Tissue and Osseous Impingement}

Although it is less commonly indicated, elbow arthroscopy can be used for the treatment of soft tissue and bony pathology that is not secondary to fracture or contracture. Arthroscopy can be performed diagnostically, and the range of motion, assessed under direct visualization to evaluate symptomatic bony or soft tissue pathology. Arthroscopic soft tissue and bony debridement or resection in the pediatric population has limited data, but studies $2,9,10,42,43$ have shown successful outcomes in the treatment of synovitis, most commonly secondary to hemarthrosis in hemophilic patients, as well as in the treatment of posteromedial impingement.

In the earliest retrospective review of pediatric elbow arthroscopy, Micheli et al. ${ }^{2}$ cited synovitis (10\%) and impingement (5\%) among the most common diagnoses and indications for arthroscopic treatment. Those with synovitis underwent arthroscopic debridement or biopsy of joint synovitis, and those with impingement underwent arthroscopic resection of olecranon fossa spurs for treatment of posterior olecranon impingement syndrome. There were no complications observed in either group, and they were among the $85 \%$ of patients reported to have good or excellent results.

The majority of the remaining literature on the pediatric population focuses on arthroscopic treatment of posteromedial impingement or valgus extension overload in throwing athletes. In a recent study, Matsuura et al. ${ }^{10}$ reported good results after arthroscopic debridement of posteromedial synovitis and excision of olecranon spurs in
15 adolescent baseball players with posteromedial elbow impingement. Though 2 patients demonstrated a recurrence of olecranon osteophytes and subsequent mild discomfort with activity, all patients were able to return to the previous level of play without complications. ${ }^{10}$ Similarly, Park et al. ${ }^{9}$ presented a series of 17 patients who underwent arthroscopic olecranon resection with or without medial collateral ligament reconstruction with symptoms of posteromedial impingement. They used MRI to evaluate the integrity of the ulnar collateral ligament (UCL), and four patients underwent olecranon resection with staged UCL reconstruction. Postoperatively, the mean range of motion with extension and supination was significantly improved; flexion and pronation were unchanged. Patients reported less pain and $85 \%$ of patients returned to play. While posteromedial impingement syndrome is less common in this population, these studies ${ }^{2,9,10}$ show that arthroscopic resection of olecranon osteophytes and removal of loose bodies have favorable outcomes.

Additionally, arthroscopic synovectomy is proving to be an excellent alternative to open synovectomy in children with recurrent hemarthrosis secondary to hemophilia. ${ }^{42,43}$ Dunn et al. ${ }^{43}$ reviewed 44 patients that underwent arthroscopic synovectomy of various joints after failed medical management, and performed arthroscopic synovectomies on 21 elbows. In those with more advanced arthritic disease, arthroscopic synovectomy was performed in conjunction with chondral debridement and osteophyte resection. Despite the lack of clinically significant improvement in elbow range of motion at 1 and 6 years of follow up, arthroscopic synovectomy decreased the rate of hemarthrosis recurrence, and there were no complications from the procedure. $^{43}$

\section{Conclusions}

Arthroscopy can treat a wide range of pediatric elbow pathologies with equal, if not better, efficacy and safety than open surgery. Arthroscopy can be considered another surgical approach, and its utility can be applied to the treatment of various disorders, including contracture releases, fracture fixation, OCD lesions, deformity corrections, and debridement of soft tissue and bony impingements. In cases of elbow contracture release, the morbidity of open surgery may preclude the ability to perform staged procedures; in this situation, arthroscopy provides the unique advantage of enabling multiple staged interventions with minimal morbidity to the patient. In trauma scenarios such as the fixation of lateral condyle fractures, the direct visualization under magnification provided by an arthroscopic approach facilitates a more anatomic reduction of the fracture with minimal risk of avascular necrosis that can occur from open procedures. As the instrumentation and techniques improve, arthroscopy should be considered a valuable tool in the surgeon's armamentarium to treat pediatric elbows. Future research is needed to address the expanding indications for pediatric elbow arthroscopy. 


\section{Conflict of Interests}

The authors have no conflict of interests to declare.

\section{References}

1 Burman MS. Arthroscopy or the direct visualization of joints: an experimental cadaver study. 1931. Clin Orthop Relat Res 2001; (390):5-9

2 Micheli LJ, Luke AC, Mintzer CM, Waters PM. Elbow arthroscopy in the pediatric and adolescent population. Arthroscopy 2001;17 (07):694-699

3 Vavken P, Müller AM, Camathias C. First 50 Pediatric and Adolescent Elbow Arthroscopies: Analysis of Indications and Complications. J Pediatr Orthop 2016;36(04):400-404

4 Kelly EW, Morrey BF, O'Driscoll SW. Complications of elbow arthroscopy. J Bone Joint Surg Am 2001;83(01):25-34

5 Marti D, Spross C, Jost B. The first 100 elbow arthroscopies of one surgeon: analysis of complications. J Shoulder Elbow Surg 2013; 22(04):567-573

6 Intravia J, Acevedo DC, Chung WJ, Mirzayan R. Complications of Elbow Arthroscopy in a Community-Based Practice. Arthroscopy 2020;36(05):1283-1290

7 Andelman SM, Meier KM, Walsh AL, Kim JH, Hausman MR. Pediatric elbow arthroscopy: indications and safety. J Shoulder Elbow Surg 2017;26(10):1862-1866

8 Hausman MR, Qureshi S, Goldstein R, et al. Arthroscopicallyassisted treatment of pediatric lateral humeral condyle fractures. J Pediatr Orthop 2007;27(07):739-742

9 ParkJY, Yoo HY, Chung SW, et al. Valgus extension overload syndrome in adolescent baseball players: clinical characteristics and surgical outcomes. J Shoulder Elbow Surg 2016;25(12):2048-2056

10 Matsuura T, Iwame T, Suzue N, et al. Clinical Outcome of Arthroscopic Treatment for Posteromedial Elbow Impingement in Adolescent Baseball Players. Arthroscopy 2018;34(01):105-110

11 Kang MS, Alfadhil RA, Park SS. Outcomes of arthroscopy-assisted closed reduction and percutaneous pinning for a displaced pediatric lateral condylar humeral fracture. J Pediatr Orthop 2019;39 (07):e548-e551

12 Yoo JS, Kim SJ, Jung JU, Seo JB. Proper elbow arthroscopy portal placement in pediatric and adolescent patients. J Orthop 2018;15 (02):455-458

13 Fink Barnes LA, Parsons BO, Hausman M. Arthroscopic management of elbow fractures. Hand Clin 2015;31(04):651-661

14 Mih AD, Wolf FG. Surgical release of elbow-capsular contracture in pediatric patients. J Pediatr Orthop 1994;14(04):458-461

15 Stans AA, Maritz NG, O'Driscoll SW, Morrey BF. Operative treatment of elbow contracture in patients twenty-one years of age or younger. J Bone Joint Surg Am 2002;84(03):382-387

16 Kodde IF, van Rijn J, van den Bekerom MPJ, Eygendaal D. Surgical treatment of post-traumatic elbow stiffness: a systematic review. J Shoulder Elbow Surg 2013;22(04):574-580

17 Gouveia K, Zhang K, Kay J, et al. The use of elbow arthroscopy for management of the pediatric elbow: a systematic review of indications and outcomes. Arthroscopy 2021;S0749-8063(21): 00064-00065

18 Nowotny J, Löbstein S, Biewener A, Fitze G, Kasten P. Elbow arthroscopy in children and adolescents: analysis of outcome and complications. Eur J Med Res 2018;23(01):42-50

19 Andelman SM, Walsh AL, Sochol KM, Rubenstein WM, Hausman MR. Arthroscopic contracture release in the pediatric patient. J Pediatr Orthop 2018;38(09):e507-e513

20 Ackerson R, Nguyen A, Carry PM, Pritchard B, Hadley-Miller N, Scott F. Intra-articular radial head fractures in the skeletally immature patient: complications and management. J Pediatr Orthop 2015;35(05):443-448
21 Koehler SM, Sakamoto S, Abernathie BL, Hausman MR. Arthroscopic correction of a supracondylar malunion in a child. Arthrosc Tech 2015;4(03):e215-e221

22 Perez Carro L, Golano P, Vega J. Arthroscopic-assisted reduction and percutaneous external fixation of lateral condyle fractures of the humerus. Arthroscopy 2007;23(10):1131.e1-1131.e4

23 Temporin K, Namba J, Okamoto M, Yamamoto K. Diagnostic arthroscopy in the treatment of minimally displaced lateral humeral condyle fractures in children. Orthop Traumatol Surg Res 2015;101(05):593-596

24 Foster DE, Sullivan JA, Gross RH. Lateral humeral condylar fractures in children. J Pediatr Orthop 1985;5(01):16-22

25 Shaerf DA, Vanhegan IS, Dattani R. Diagnosis, management and complications of distal humerus lateral condyle fractures in children. Shoulder Elbow 2018;10(02):114-120

26 Mirsky EC, Karas EH, Weiner LS. Lateral condyle fractures in children: evaluation of classification and treatment. J Orthop Trauma 1997;11(02):117-120

27 Marzo JM, d'Amato C, Strong M, Gillespie R. Usefulness and accuracy of arthrography in management of lateral humeral condyle fractures in children. J Pediatr Orthop 1990;10(03): 317-321

28 Murthy PG, Vuillermin C, Naqvi MN, Waters PM, Bae DS. Capitella fracture in children and adolescents: classification and early results of treatment. J Bone Joint Surg Am 2017;99(15): 1282-1290

29 Kim HT, Lee JS, Yoo CI. Management of cubitus varus and valgus. J Bone Joint Surg Am 2005;87(04):771-780

30 Miyake J, Masatomi T. Arthroscopic debridement of the humeral capitellum for osteochondritis dissecans: radiographic and clinical outcomes. J Hand Surg Am 2011;36(08):1333-1338

31 Jones KJ, Wiesel BB, Sankar WN, Ganley TJ. Arthroscopic management of osteochondritis dissecans of the capitellum: midterm results in adolescent athletes. J Pediatr Orthop 2010;30 (01):8-13

32 Takeba J, Takahashi T, Watanabe S, et al. Short-term clinical results of arthroscopic osteochondral fixation for elbow osteochondritis dissecans in teenaged baseball players. J Shoulder Elbow Surg 2015;24(11):1749-1756

33 Tis JE, Edmonds EW, Bastrom T, Chambers HG. Short-term results of arthroscopic treatment of osteochondritis dissecans in skeletally immature patients. J Pediatr Orthop 2012;32(03): 226-231

34 Labelle H, Bunnell WP, Duhaime M, Poitras B. Cubitus varus deformity following supracondylar fractures of the humerus in children. J Pediatr Orthop 1982;2(05):539-546

35 Hyatt BT, Schmitz MR, Rush JK. Complications of Pediatric Elbow Fractures. Orthop Clin North Am 2016;47(02):377-385

36 Rubin G, Orbach H, Bor N, Rozen N. Tardy Ulnar Nerve Palsy. J Am Acad Orthop Surg 2019;27(19):717-725

37 Abe M, Ishizu T, Morikawa J. Posterolateral rotatory instability of the elbow after posttraumatic cubitus varus. J Shoulder Elbow Surg 1997;6(04):405-409

38 Davids JR, Maguire MF, Mubarak SJ, Wenger DR. Lateral condylar fracture of the humerus following posttraumatic cubitus varus. J Pediatr Orthop 1994;14(04):466-470

39 Takahara M, Sasaki I, Kimura T, Kato H, Minami A, Ogino T. Second fracture of the distal humerus after varus malunion of a supracondylar fracture in children. J Bone Joint Surg Br 1998;80(05): 791-797

40 Solfelt DA, Hill BW, Anderson CP, Cole PA. Supracondylar osteotomy for the treatment of cubitus varus in children: a systematic review. Bone Joint J 2014;96-B(05):691-700

41 Oppenheim WL, Clader TJ, Smith C, Bayer M. Supracondylar humeral osteotomy for traumatic childhood cubitus varus deformity. Clin Orthop Relat Res 1984;(188):34-39 
42 Journeycake JM, Miller KL, Anderson AM, Buchanan GR, Finnegan M. Arthroscopic synovectomy in children and adolescents with hemophilia. J Pediatr Hematol Oncol 2003;25(09):726-731
43 Dunn AL, Busch MT, Wyly JB, Sullivan KM, Abshire TC. Arthroscopic synovectomy for hemophilic joint disease in a pediatric population. J Pediatr Orthop 2004;24(04):414-426 